\title{
Reaching Universal Health Coverage by 2035: Is Cameroon on Track?
}

\author{
Chenjoh Joseph Nde ${ }^{1, *}$, Alice Raymond ${ }^{2}$, Yauba Saidu ${ }^{3}$, Ndong Ignatius Cheng ${ }^{4}$, Divine Nzuobontane ${ }^{3}$, \\ Johannes Tabi Atemnkeng, ${ }^{5}$, Wilfred Fon Mbacham ${ }^{6}$

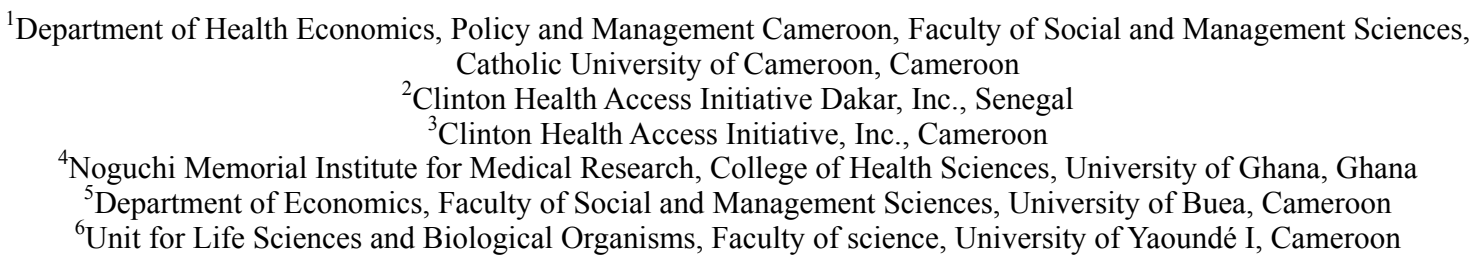

Copyright $(2019$ by authors, all rights reserved. Authors agree that this article remains permanently open access under the terms of the Creative Commons Attribution License 4.0 International License

\begin{abstract}
Access to health care is a fundamental human right, which places a legal obligation on states to ensure access to quality, timely, acceptable, and affordable health care for its citizens. However, many states struggle to meet this basic right for their citizens given that every year about 100 million people are pushed into poverty, while 150 million people suffer financial catastrophe due to out of pocket payments for health. In Cameroon, studies have shown that about $64 \%$ of households cannot access healthcare, because they perceive the cost of care to be high. In 2012, the National Health Accounts (NHA) estimated that about $70 \%$ of the Total Health Expenditure (THE) for the country was borne by households. Compared to its peers, the contribution of Cameroonian households is the third highest in Sub-Saharan Africa, where the average is 34\%. To remedy this situation, Cameroon has initiated reflections aimed at establishing a universal health coverage (UHC) scheme for its population. This paper therefore aimed at providing a synthesis of Cameroon's progress and assessing if the country is on track to achieving UHC by 2035.
\end{abstract}

Keywords Universal Health Coverage, Benefit Package, Costing, Matriculation

\section{Introduction}

Access to health care is a fundamental human right, which places a legal obligation on states to ensure access to timely, acceptable, and affordable quality health care for its citizens. [1] Yet, many governments, struggle to meet this basic right for their citizens as estimates suggest that globally about 400 million people lack access to essential health services while 8 million die from treatable medical conditions leading to yearly losses of 6 trillion USD in economic welfare for low- and middle-income countries. [2] Each year, about 100 million people are pushed into poverty while 150 million people suffer financial catastrophe because of out of pocket payments for health services. [3]

To reverse this trend, world leaders adopted the Sustainable Development Goals (SDGs) in 2012. In sub goal 3.8, they committed their governments to attain universal health coverage (UHC) including financial risk protection, access to quality and affordable essential medicines and vaccines for all. [4] Many African countries have integrated UHC as a goal in their national health strategies. In spite of this, progress in translating these commitments into expanded domestic resources for health, effective development assistance, and ultimately, equitable and quality health services with increased financial protection, has been slow [5]. Consequently, households continue to bare a greater burden of health expenditures in this region.

In Cameroon, for instance, about $70 \%$ of the 156 million USD total domestic health expenditure in 2012 was borne by households. This high contribution by Cameroonian households, which nearly doubles the regional average of $34 \%$, ranks Cameroon third in sub-Saharan Africa, affecting the population's access to healthcare services in the country. [6]Indeed, one report suggests that about $64 \%$ of households in Cameroon were unable to access healthcare services because they perceived the cost to be high. [7] In order to reverse this trend, the government of Cameroon has taken a number of important steps aimed at improving the access to health services for the population. Most health financing reforms 
that are currently being implemented in the country, are aimed at attaining UHC for the population by 2035. This is also clearly enshrined in national policy documents such as the Vision 2035, the Growth and Employment Strategy paper (GESP), the Health Sector Strategy (HSS2016-2027) and the National Health development Plan (NHDP)

The incidence of catastrophic payments amongst Cameroonian household is high. Indeed, $36.3 \%$ of the population spends more than $5 \%$ of their income on health while nearly $5 \%$ of the population spend above $40 \%$ of their income on health, leading to catastrophic payments. An analysis of health equity and financial protection shows that the higher the expenditures as a percentage of income, the more the poorest segments of the population are affected. This means that the poorest are the ones who suffer the most from these catastrophic health expenses in Cameroon. [8]

To remedy this situation, the government set up the National Technical Working Group for UHC (TWG UHC) to pilot discussions around the development of a UHC scheme for the country. The group, which is co-chaired by the minister of Public Health and the minister of Labor and Social Security, is made up of members drawn from government institutions, the civil society and technical and financial partners (TFP). This group is charged with coordinating national thinking and designing a roadmap for the development of the UHC in Cameroon. In this paper, we review Cameroon's experiences and choices as well as develop a normative framework of the progress the country has made towards the development of its UHC scheme.

\section{Methods}

The multiplicity of actions for the development of the UHC, resulted to parallel outputs on UHC development in Cameroon. A series of independent activity reports, studies, workshop presentations, policy proposals and key documents were produced by various structures and organizations directly implicated in the development of the UHC in Cameroon. This often-disconnected reports and activities have made it difficult for a comprehensive picture and assessment of progress towards UHC to be done. In order to build a comprehensive picture of Cameroon's progress, we contacted a cross section of stakeholders and rapporteurs from various sub-working groups and organizations and copies of activity reports, studies and workshop presentations on the development of the UHC in Cameroon were obtained. As a result, we generated a database of UHC related activity reports and documents for Cameroon which we reviewed for this study.

In order to provide a succinct synthesis of the country's experiences and choices, we reviewed 86 related working documents. These documents included: reports from TWGs and sub-working groups (SWGs), presentations at workshops on the development of particular aspects of the UHC and final reports following validation workshops. We then classified these documents into seven categories namely: 1) Situational analysis, 2) UHC architecture, 3) benefit package development, 4) UHC matriculation, 5) UHC legislature, 6) UHC communication strategy and; 7) other related supplementary studies. After our review, we eliminated 41 of the 86 documents because these were draft documents and activity reports of sub working groups. We also eliminated all meeting reports because final sub-working group reports were a summary of these meeting reports. Following this elimination, we retained 45 documents for our review of which, 29 were PowerPoint presentations delivered at workshops and 16 were final approved reports from sub working groups and studies. We exploited these documents in order to develop a comprehensive picture and measure the progress of Cameroon in the development of the UHC.

\section{Results}

\subsection{Steps in the Development of the UHC}

\subsubsection{Situational Analysis of the State of Health Risk Protection in Cameroon}

A situational analysis on equity in the financing of health and providing financial protection against sickness revealed that only $6.46 \%$ of Cameroon's population is covered by a community health insurance scheme. This low coverage implies that a majority of the population continue to support their health expenditure through out of pocket payments. By 2014, about 43 active mutual health organizations existed covering about 63,000 people $(0.2 \%$ of the population) with contributions ranging from 6 to 10 USD per person per year. However, the contribution of these insurance schemes to poverty reduction by significantly reducing out of pocket payments and contributing to the total health expenditure remains negligible. [9]

In 2012, the prevalence of catastrophic health expenditure ranged from 4.7 to 21 percent of households.[10]Health expenditures increased the poverty rate by almost 2 percentage points, and exacerbated the depth of poverty by almost 12 percentage points in Cameroon. Consequently, reducing out-of-pocket health spending will directly reduce both the incidence and severity of poverty in the country. [11] The private sector as well has been keen on addressing this problem. A survey in 2014 revealed that, 16 private health insurance companies exist, providing coverage to approximately 190,408 persons. However, their impact on poverty reduction equally remains minimal, given the high annual subscription cost ranging from USD 310 per adult to USD 
150 per child, which automatically excludes the poor who are most in need of health insurance coverage.[9]

The state in the past has attempted at providing financial risk protection against the risk of ill health within the framework of the National Social Insurance Fund (NSIF). Affiliation to the NSIF is mandatory for employees of the private formal sector and voluntary for those of the informal sector. The NSIF runs two main aspects of financial risk protection for workers, which include social protection and occupational disease and accident schemes. In 2010, 4074 beneficiaries were part of the occupational disease and accident scheme and 368,455 beneficiaries for the social benefits arm. However, the population coverage by the NSIF remains negligible with just about $3.2 \%$ of the total population covered. [9]

\subsubsection{UHC Guiding Principles for Cameroon}

Reflections on the design of a UHC scheme for Cameroon centered on the three dimensions of UHC. These dimensions include: i) How to expand the service package to include more services; ii) How to expand coverage to cover the entire population and iii) How to reduce out of pocket payments. In order to adequately address each dimension, experts identified and adopted four basic principles to guide the development of the UHC in Cameroon. These included:

1) Universality: All sections of the population, without any distinction, are entitled to a basic package of services. This basic package should ensure that all those who need protection against the risks associated with a disease are able to access quality care without barriers.

2) National solidarity: Each individual should contribute according to his/ her capacity but is entitled to the same quantity of benefits in the event of illness (equity in contribution and access to care).

3) General responsibility of the State: Health is a public good and social protection is a right enshrined in the constitution, therefore, the attainment of good health is a major responsibility of the state.

4) Mandatory affiliation: In accordance with the notions of solidarity and universality, extending coverage to all will be done on the basis of compulsory affiliation. [12]

The underlying rational for adopting these basic guiding principles is to ensure equity in access to health care and use of services, guarantee the quality of services provided, offer coverage for all and above all provide financial protection against the risk of ill health. The daunting challenge will be the implementation of the obligatory principle especially within a Cameroonian context where a great majority of the population operates in the informal sector. However, experts are already envisaging the use of a combination of mechanisms adapted to the mode, organization and functioning of the
Cameroonian informal sector in order to mitigate this threat.

\subsubsection{Cameroon's UHC Architecture}

In October 2016, a UHC architecture was designed and proposed by the UHC-TWG. The architecture proposes the establishment of a single national structure for the technical and financial management of UHC in Cameroon. This institution shall have the status of a public enterprise with administrative and financial autonomy. [13]

The existence of different categories of the population dictates that for each category, specific adapted mechanisms will be put in place to facilitate enrollment and collection of premiums. Consequently, there will be a delegation of the affiliation and premium collection responsibilities to specific organizations such as the Regional Special Funds for Health Promotion (RSFHP) and municipal authorities. Although the role of the state as guarantor of social security and health of the population cannot be disputed, there is a need for transparency and accountability in the management of the UHC. Consequently, all stakeholders particularly the beneficiaries and civil society would be involved in the management structure of the UHC scheme.

\subsubsection{The UHC Benefit Package}

The development of the UHC benefit package was done following 2 stages. For stage 1, the main focus was to include a great majority of medical conditions that have a great impact on the health of the population. As a result, this led to the identification of 3,416 diseases or medical conditions. This list of interventions was subsequently adjusted to meet the health needs of children less than five, adolescents and pregnant women. As a result, 666 medical conditions or diseases were retained and were grouped into 440 interventions for the minimum package of interventions and 226 interventions constituting a complimentary package of interventions. [14]

In stage 2, the focus was to develop a list of interventions that is not only affordable but is also cost effective. As such, the lists of 3,416 diseases having an impact on the health of the population were classified in terms of their associated Disability Adjusted Life Years (DALYs) and mortality rate. This led to the identification of 959 conditions based on: 1) the frequency of the condition; 2) the possibility of the condition being treated by a general practitioner at the level of the integrated health center (IHC), the medicalized health center(MHC) and the District Hospital (DH) and 3) priority community activities. In the end, 183 medical conditions were retained, which included 48 (26.37\%) interventions for children, 25 (13.74\%) interventions for pregnant women (73 interventions for both mother and child) and 109 interventions to carter for the needs of the entire population $(59.89 \%)$. [15] 


\subsubsection{Development of the Treatment Protocols for the UHC Benefit Package}

After the development of the list of interventions, medical specialists were brought together to develop the treatment protocol for each medical condition. The exercise facilitated the definition of the following: a clear definition of the condition, the epidemiological elements (prevalence, incidence, mortality), the clinical manifestations of the condition, and a list of all the inputs necessary to deliver the required curative services.[16] This exercise facilitated the development of the standard treatment protocols for each of the 183 medical conditions. These protocols will serve as a means of ensuring the provision of standardized quality medical care services throughout the national territory irrespective of the type of health facility (private, public or confessional) and the geographical location of the health facility. The treatment protocols were developed using the syndromic approach, as defined in the "National Standard Diagnostic and Therapeutic Guidelines for Primary and Intermediate Medical Care" published in 2016 by the $\mathrm{MoH}$.

\subsubsection{Costing of the UHC Treatment Package}

Once the treatment protocols were developed, experts including pharmacists, health economists, civil society actors and medical practitioners came together to generate the cost(unit and total cost) of treating a patient for each medical condition included in the benefit package. The costing exercise was guided by the following assumptions:

1. The calculated costs would serve as a basis for negotiations on reimbursements of care services provided by public and private health facilities;

2. Costing would include only direct costs such as the cost of drugs and consumables and would exclude indirect costs such as wages, utilities (water and electricity) and the depreciation of equipment and infrastructure;

3. Public health services that are currently offered for free through subsidized health programs would continue to be provided by the government and its technical and financial partners (TPF) and will not be included in the UHC. This assumption caused the list of interventions to drop from 183 to 150 medical conditions;

4. The benefit package will be provided over a time horizon of 10 years, with 2018 serving as the base year;

5. The proportion of the population using the services may vary after the analysis due to an increase in enrollment or by an increased use of services by existing members;

6. Unit costs may vary from year to year depending on factors such as inflation, increased use of generic pharmaceuticals.

7. Direct unit costs were assumed to be the same for both public and private providers.

Guided by these assumptions, the team of experts estimated the cost of providing 150 interventions over the next ten years. The costed interventions were classified under 10 thematic areas. Data for the unit cost of inputs for each intervention were obtained from decree No. 87-529 of $21^{\text {st }}$ April 1987, which established the prices of services for medical and paramedical procedures. Once the cost of treating one patient was generated, the cost of treating the estimated number of persons expected to suffer from the disease in a year was generated by multiplying the cost per patient by the prevalence of the disease. Table 1 below presents a projection of the cost of the benefit package over the next 5 years. The exercise estimated that it would require the sum of USD 2.8 billion to provide the interventions included in the UHC package to the entire population.

Table 1. Estimated cost of providing the 183 interventions included in the proposed UHC benefit package from 2018 to 2022 (values in millions of USD)

\begin{tabular}{|l|r|r|r|r|r|}
\hline \multirow{2}{*}{ Benefits package } & \multicolumn{5}{c|}{ Cost } \\
\cline { 2 - 6 } & \multicolumn{1}{|c|}{$\mathbf{2 0 1 8}$} & $\mathbf{2 0 1 9}$ & \multicolumn{1}{c|}{$\mathbf{2 0 2 0}$} & \multicolumn{1}{c|}{$\mathbf{2 0 2 1}$} & $\mathbf{2 0 2 2}$ \\
\hline Internal medicine & $1,091.5$ & 1075.7 & 1114.5 & 1154.9 & 1197.6 \\
\hline Pediatrics & 433.2 & 438.2 & 442.3 & 446.8 & 451.6 \\
\hline Management & 410.0 & 413.1 & 425.0 & 43.7 & 450.0 \\
\hline Gynecology & 282.7 & 290.6 & 299.4 & 308.4 & 317.7 \\
\hline Surgery & 158.8 & 175.0 & 180.2 & 185.6 & 191.2 \\
\hline Ophthalmology & 73.2 & 75.6 & 78.1 & 80.9 & 83.8 \\
\hline Stomatology & 65.6 & 67.6 & 69.7 & 71.9 & 74.1 \\
\hline Vaccination & 65.9 & 63.3 & 64.7 & 66.1 & 67.7 \\
\hline Oto-Rhino-Laryngology & 55.4 & 56.0 & 56.7 & 57.4 & 58.0 \\
\hline Community Health & 5.9 & 4.8 & 5.7 & 4.8 & 5.9 \\
\hline Total & $\mathbf{2 , 7 3 3 . 6}$ & $\mathbf{2 , 7 5 3 . 9}$ & $\mathbf{2 , 8 3 3 . 2}$ & $\mathbf{2 , 9 1 3 . 5}$ & $\mathbf{3 , 0 0 0 . 2}$ \\
\hline
\end{tabular}

Source: Health Financing and Governance. 2017. Pre-validation of data for the cost estimate of the UHC benefit package for Cameroon 


\subsubsection{Development of the UHC Matriculation System}

Cameroon has opted for a biometric matriculation system for the registration of its population into the UHC scheme. This option was preferred because of its ability to minimize fraud which is a major challenge facing most health insurance schemes. Biometric registration directly ties with the vision of the $\mathrm{MoH}$ to gradually transitioning from paper-based health records to a system of electronic medical records. The proposed system will require the collection of three broad sets of information from each adherent during the registration process. These include:

- Biographical and biometric data: Biographical data will consist of the names, date and place of birth, sex, photograph, parents ' names and professional status of the adherent. In addition, the following biometric parameters of the adherent will also be collected: fingerprints, iris, facial recognition, voice stamp etc.

- Data from other registration systems, such as the national identity card number, birth certificate number, passport number, national disability card number for socially vulnerable persons, residence permit number for foreign nationals, NSIF number and telephone numbers from mobile providers;

- Additional information will be collected from the adherent on the council area where the biometric card will be delivered, email, town of residence, neighborhood, street, post box number, name of employer and full address of the employer. [17]

Every adherent will be issued a unique identification number, comprising of 13 digits using the following format: Year and Month - Municipality - Number Key. [17] The number will be generated as follows:

- Year: Which will be the last two digits of the year at the time of enrollment;

- Month: Numeric value of the month at the time of enrollment, numbered from 01 to 12 .

- Municipality: A unique identifier of the municipality codified in 4 digits. Possible values will range from 001 to 9999 .

- Serial number: A 4 digits coded serial number of the beneficiary enrolled in the municipality for the year and month concerned with a maximum value of 9999 per month.

- Key: A conformance control key for checking the authenticity of the previous numbers, which will be calculated using LUHN algorithm.

Apart from simply serving as a registration system, the proposed biometric system will be highly integrated and will be used for managing the payment of contributions, processing the reimbursement of services provided and for auditing the entire UHC system. The government equally hopes to leverage on this system to embark on a general overhaul of the health information management system.

\subsubsection{Determination of Contributive Capacity of the} Population and the Startup Benefit Package

After the costing of the benefit package, reflections shifted to mobilizing the necessary finance for the UHC scheme. This led to a study on the classification of the population according to socio-economic factors. [18] This study classified the population into five socio-economic groups (Inactive, informal agricultural sector, informal non-agricultural sector, formal private sector, and workers of the public and para-public sectors) and estimated the contributive capacity of each group to the UHC scheme. According to the study, the highest contributors to the UHC will be workers of the public and para-public sectors while the inactive population will constitute the lowest contributors to the system.

Table 2.Classification of the population according to socio-economic characteristics and determination of the contributive capacity

\begin{tabular}{|l|l|r|}
\hline No & Socio-economic classification & $\begin{array}{r}\text { Contributory } \\
\text { capacity (USD) }\end{array}$ \\
\hline 1 & Inactive population & 38 \\
\hline 2 & Informal agricultural sector & 47 \\
\hline 3 & Informal non-agricultural sector & 100 \\
\hline 4 & $\begin{array}{l}\text { Employees of the formal private } \\
\text { sector }\end{array}$ & 110 \\
\hline 5 & $\begin{array}{l}\text { Employees of the public and } \\
\text { para-public sector }\end{array}$ & 57 \\
\hline
\end{tabular}

Source: ILO. 2017. Elaboration of a classification of the Cameroonian population according to socio-economic characteristics and the determination of contributory capacities for universal health coverage

Using income data from 2014, it was estimated that nearly 8 million USD would be generated from the five socio-economic classes. However, this amount is less than $1 \%$ of the current contribution of households to the total health expenditure of the country, which is currently estimated at about 1.03 billion USD. [18]

The high cost of providing the benefit package amidst fiscal constraints and a weak contributive capacity of the population, made it imperative for a more affordable reduced UHC benefit package to be developed. For this reason, there was a need for the target population to be redefined based on service need, mortality rate and population coverage. As a result, a new reduced benefit package, containing 85 medical interventions was deduced from the initial list of 183. Table 3 below presents the set of services included and the estimated cost of providing the services for the year 2019. It is estimated that this reduced start up benefit package will require the sum of about USD 600 million to be fully implemented

Based on this list of 85 medical conditions, the government plans to break up the implementation of the UHC scheme into three phases. Each phase will cover a specified period and will be tied to a particular objective as shown on table 4 below. 
Table 3. Estimated cost of implementing the first phase of the reduced UHC benefit package (in millions of USD)

\begin{tabular}{|l|c|}
\hline \multicolumn{1}{|c|}{ Healthcare services } & Cost per year \\
\hline Pediatrics & 287.8 \\
\hline Gynecology/obstetrics & 190.7 \\
\hline HIV AIDS. Malaria. Tuberculosis. Cancer. Dialysis. Schistosomiasis. intestinal helminthiasis & 122.9 \\
\hline Vaccinations & 63.3 \\
\hline Health promotion services (Community health) & 4.8 \\
\hline Total cost of the first phase & $\mathbf{6 6 9 . 5}$ \\
\hline
\end{tabular}

Source: adapted from Cheumaga. 2018. Targets, subsidized health care services and cost. A PowerPoint presentation at the validation workshop of the first phase of the UHC.

Table 4.Phases of the implementation of the UHC in Cameroon

\begin{tabular}{|l|l|l|c|}
\hline \multicolumn{1}{|c|}{ Phases } & Period & \multicolumn{1}{|c|}{ Objective } & $\begin{array}{c}\text { Enrollment } \\
\text { target }\end{array}$ \\
\hline Phase 1 & 2 years & Establishing the structures, instruments and tools for the management of the UHC system. & $30 \%$ \\
\hline Phase 2 & 5 years & Attaining full coverage of the initial benefit package of 85 interventions. & $60 \%$ \\
\hline Phase 3 & 5 years & Expansion of both the benefit package to include all 185 interventions and the target population. & $100 \%$ \\
\hline
\end{tabular}

Source: Adapted from Longang E. V. Synthesis of UHC work in Cameroon.2018. PowerPoint presentation at the workshop on the UHC between the government and technical and financial partners. Yaounde, Cameroon

The enrollment into the UHC will be progressive and based on pre-defined yearly coverage targets. However, current ongoing discussions seem to indicate that the government intends to embark on a national roll out of the scheme i.e. begin the UHC in every district of the country at the same time.

\section{Discussion}

The main purpose of this paper was to provide a synthesis of Cameroon's choices and to assess if the country is on track to developing a UHC scheme by 2035. To this effect, a normative framework of the countries progress is shown on figure 1 below. The figure breaks down the progress towards UHC into 3 main stages (with each stage depending on the previous stage) consisting of 13 steps and sub processes. While all stages have been developed sufficiently, stage 3 is still to be completely addressed.

Universal health coverage implies examining two major components of the health system; 1) Strengthening the supply of care by ensuring the availability of quality health care services and 2) organizing the financing of the demand for health care. A situational analysis of the development of the UHC reveals that a lot has been achieved in organizing the demand for health care while much still has to be done to reorganize the supply side. A major problem facing the supply of health care in Cameroon is the acute shortage of qualified personnel, which is aggravated by geographic distributional inequalities of the national health workforce and the absence of basic infrastructure and equipment in health facilities. [19]Consequently, this has resulted to a consistent fall in the standards of care in public health facilities throughout the country. [20] Given that UHC will lead to an upsurge in the demand for healthcare throughout the country, considerable efforts will have to be invested in addressing the distributional inequalities of qualified health personnel and upgrade the technical platform of public and private health facilities so as to ensure a uniform level of service availability and readiness across all health districts before the eventual roll out of the UHC scheme.

The separation of the buyer and supplier functions in the UHC is crucial to ensuring a sustainable long-term provision of quality services. The proposed UHC architecture clearly states that the national health insurance institution will be an autonomous public enterprise separate from the $\mathrm{MoH}$ who is the major supplier of health care services. In this way, the buyer will be able to hold the supplier accountable and push for a continuous improvement in the quality of services provided. It is expected that the level of efficiency in the health system will also generally increase and the population will benefit from better quality services at an affordable price. The proposed architecture also suggests the putting in place of a single funding pool. This is in direct contrast to what obtains in Rwanda and Gabon wherein different funding pools exist for different categories of the population. [21] It is argued that the putting in place of a single funding pool will facilitate cross subsidization and reinforce the concept of national solidarity, which is a major guiding principle of the Cameroonian UHC. 


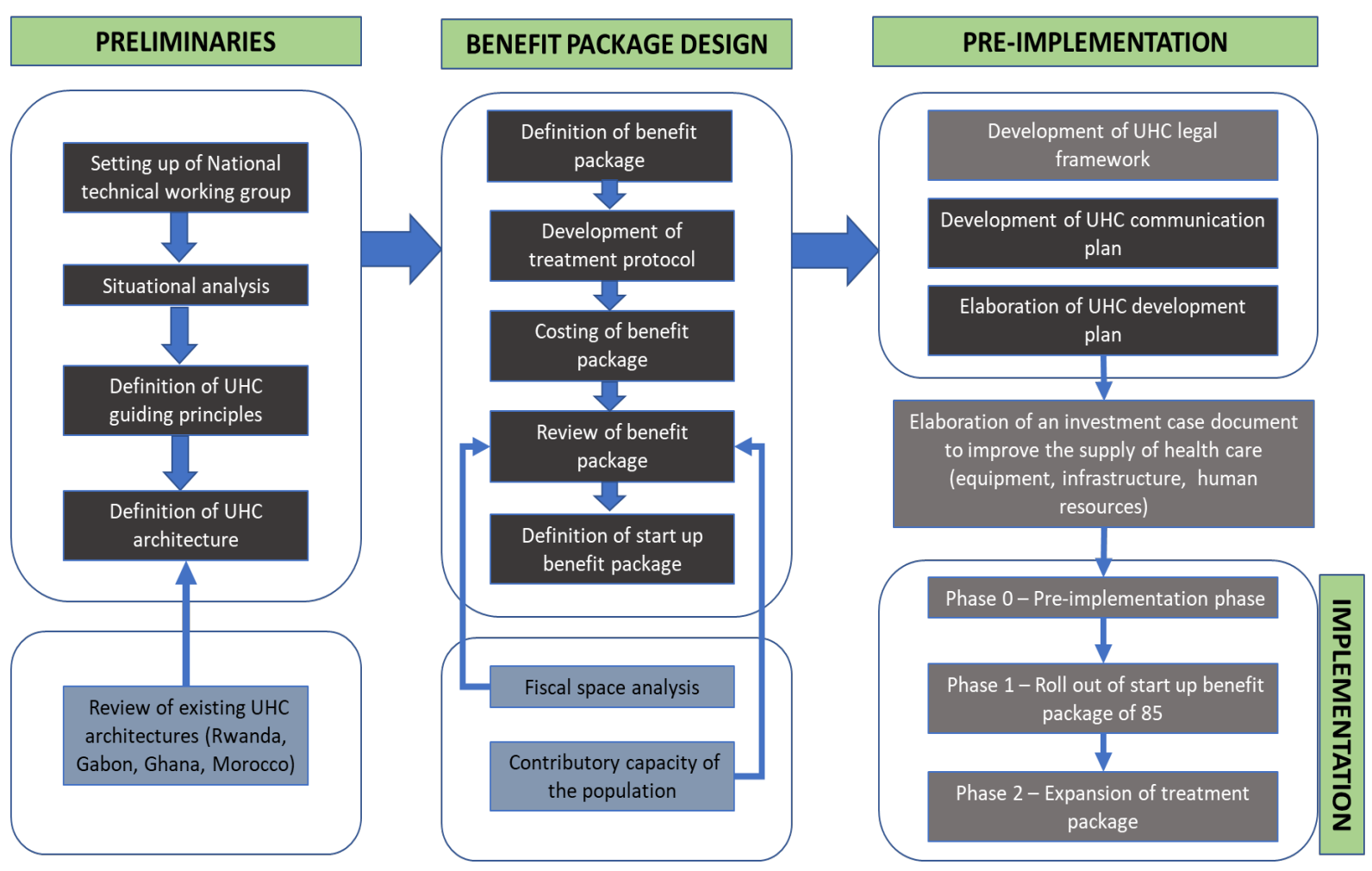

Figure 1. Normative framework of the development of the UHC in Cameroon

The costing of the benefit package suffers from a serious limitation. The prices used for estimating the cost of all inputs required for each intervention is based on prices that were homologated in 1987 i.e. 30 years back. This is because since 1987, there has been no subsequent decree to regulate and update the prices of medical inputs and services to reflect the rapidly changing market trends. It is most likely that these prices do no longer reflect the current market prices for medical inputs in the country and so may constitute a major hold up to the smooth operation of the UHC as the actual cost of medical inputs may be higher than estimated when using current market prices.

The development of the UHC benefit package was an iterative process. This is because Cameroon had to strike a balance between providing as much coverage in terms of the size of the benefit package and the affordability of the package. In order to achieve this, the country defined the most vulnerable populations in need of health services and based on this, it developed a startup list of 85 interventions.

Given that the implementation of the UHC is a totally new experience for the country, opting to begin with a roll out in all districts, is obviously over ambitious for a country with a weak health system. Starting small perhaps through a pilot in some key health districts will facilitate rapid learning, gradual reinforcement of capacities and incorporation of lessons learnt into the system. This will be vital in building a robust UHC for Cameroon. In addition, the country will have to borrow lessons from other peers who are far advanced in the development of their UHC schemes. It will have to learn from Ghana, Rwanda, Kenya and Senegal on how they subsidized the enrollment of low-income groups in pre-payment mechanisms, which has significantly increased access to care. It will also have to learn from countries like Rwanda that has leveraged on its results-based financing scheme to improve the overall efficiency of its health system.[11]

Cameroon is more than half way gone into the development of its UHC scheme. Nonetheless, a key question is: when will the UHC scheme go fully operational in the country? The answer to this question, is hardly straight forward because the UHC and its accompanying health system reforms are issues with political connotations and therefore, it is risky to make a prediction on when the UHC will effectively begin. For now, what is important is to maintain a sustained high political engagement across government and to develop a clear roadmap, which clearly defines all aspects that need to be addressed before the system can go operational. In this light, some key issues which still have to be addressed include: reinforcing the supply of healthcare, finalizing the development of the health financing strategy, defining an accreditation system for health facilities, producing the tools and procedures necessary for managing benefits and controlling health expenditures as well as putting in place an information management system for the UHC.

\section{Conclusions}

After four years of participatory reflection, the National 
Technical Working Group for the UHC has made laudable progress in defining the basic elements necessary for political decision-making on the implementation of the UHC. The set of elements proposed are based both on lessons learned from international experiences on UHC development and the Cameroonian experience with community health organizations. If the current pace and high level of political engagement is sustained, Cameroon stands a chance of developing a UHC scheme for its population by 2035 .

\section{Acknowledgements}

We thank our colleagues from the government of Cameroon and members of the National Technical Working Group for the UHC who provided insight and expertise that greatly assisted the research, although they may not agree with all of the interpretations/conclusions of this paper.

This article was supported in part through WFM by the DELTAS Africa Initiative. The DELTAS Initiative is an independent funding scheme of the African Academy of Sciences (AAS)'s Alliance for Accelerating Excellence in Science in Africa (AESA) and supported by the New Partnership for Africa's Development Planning and Coordination Agency (NEPAD Agency) with funding from the WELLCOME Trust [10774/A/15/Z of March 30, 2016] and the UK government. The views expressed in this publication are those of the author(s) and not necessarily those of AAS, NEPAD Agency, Welcome Trust or the UK government.

\section{Disclaimer}

The views and opinions expressed in this article are those of the authors and do not necessarily reflect the official policy or position of any of the organisations for which they work or the government of Cameroon.

\section{REFERENCES}

[1] WHO. Human rights and health. 2017. [accessed 2018 Aug 21 ]. http://www.who.int/news-room/fact-sheets/detail/hum an-rights-and-health

[2] Harvard Medical School. Preventable deaths from lack of high-quality medical care cost trillions. 2018. Science Daily. [accessed 2018 Sept 11].www.sciencedaily.com/releases/2 018/06/180604160447.htm

[3] WHO, Universal Health Coverage, Fact sheet. [accessed 2018 Aug 22]. https://afro.who.int/health-topics/universalhealth-coverage

[4] The United Nations. Transforming our world: The 2030 agenda for sustainable development. [accessed 2018 Sept 10]. www.sustainabledevelopment.un.org

[5] World Bank. Universal health coverage in Africa: A framework for action. [accessed 2018 Sept 6]. http://www.worldbank.org/en/topic/universalhealthcoverag e/publication/universal-health-coverage-in-africa-a-framew ork-for-action

[6] Ministry of Public Health. 2012 National Health Accounts. 2016

[7] IFORD. 2015

[8] World Bank training session on ADePT analysis of data from the National Health Accounts

[9] Ministry of Public Health. OASIS report. 2016

[10] Wagstaff, et al. Health spending is defined as catastrophic when it exceeds 10 percent or 25 percent of household consumption. 2018.

[11] World Bank. Public Expenditure Review. 2018

[12] Longang E.V. Universal health coverage in Cameroon.2017. PowerPoint presentation at the annual conference of central and deconcentrated services of the ministry of health. Yaounde. Cameroon

[13] National Technical Working Group for UHC. A proposal for a UHC architecture in Cameroon. 2016. A technical Note

[14] Ministry of Health. Report of the national technical working group on the UHC treatment package for Cameroon. 2017.

[15] Ministry of Public Health, 2017, Report of the national technical working group on the UHC treatment package for Cameroon

[16] Ministry of Health, Protocol of interventions for UHC generalities. 2017. Version 2

[17] Massom S. 2018. Elaboration of a beneficiary registration methodology for Universal Health

[18] International Labour Organisation. Elaboration of a classification of the Cameroonian population according to socio-economic characteristics and the determination of contributory capacities for universal health coverage. 2017.

[19] Tandi et al. Cameroon public health sector: shortage and inequalities in geographic distribution of health personnel. 2015. International Journal for Equity in Health (2015) 14:43 DOI 10.1186/s12939-015-0172-0

[20] Manyisa Z. Mvan AEJ. 2017.Factors affecting working conditions in public hospitals: A literature review. 2017. International Journal of Africa Nursing Sciences 6 (2017) 28-38. http://dx.doi.org/10.1016/j.ijans.2017.02.002

[21] Ministry of Health. Proposal for an universal health coverage system architecture in Cameroon. 2016. National technical working group for UHC, Yaounde, Cameroon. 